\title{
Quemerina, omentina-1 y miR-103p y su relación con la diabetes mellitus gestacional
}

\author{
Chemerin, omentin-1 and miR-103p and their relationship with \\ gestational diabetes mellitus
}

\author{
Sosa García BC $C^{1}$, Mendieta Zerón $H^{2}$, Hinojosa Juárez $A C^{1}$, García $M C^{3}$
}

${ }^{1}$ Universidad Autónoma del Estado de México.

${ }^{2}$ Universidad Autónoma del Estado de México, Hospital Materno-

Perinatal "Mónica Pretelini Sáenz".

${ }^{3}$ Universidad de Santiago de Compostela, España, CITOBES

laboratorio del Centro de Investigación en Medicina Molecular y

Enfermedades Crónicas (CIMUS)/departamento de Fisiología.

Autor de correspondencia: Betsy Corina Sosa García

Correo electrónico: sosabetsy@gmail.com

Fecha de recepción: 22/08/2019

Fecha de aceptación: 10/12/2019

\section{Resumen}

Diversos genes pueden desencadenar la diabetes mellitus gestacional (DMG), considerada como un problema de salud pública de etiología compleja y multifactorial.

Objetivo: presentar una revisión de tema de la evidencia científica disponible sobre el impacto de la expresión de los genes omentina-1, quemerina y miR-1013p, implicados en el desarrollo de la DMG.

Materiales y métodos: se realizó una búsqueda de estudios electrónicos transversales a través de la base de datos registrados en MEDLINE y PUBMED, publicados durante 2010-2019, que valoraran mediciones de los tres genes en mujeres con DMG.

Resultados: ocho artículos cumplieron con los criterios de inclusión, cuatro artículos midieron los niveles de quemerina, tres de omentina-1 y uno de miR-103p. Entre los niveles de quemerina se mostró que la circulación de quemerina y miR103 p se correlacionó positivamente con las variables metabólicas durante el primer y tercer trimestre del embarazo. Se demostró que la liberación de omentina-1 fue mayor en el tejido adiposo vs. placenta cuando se presenta obesidad.

Conclusión: la expresión de genes y la presencia de obesidad están relacionados con el desarrollo de la diabetes mellitus gestacional.

Palabras clave: diabetes gestacional, genes, obesidad, tejido adiposo, glucosa.

\section{Abstract}

Several genes can trigger gestational diabetes mellitus (GDM), considered a public health problem of complex and multifactorial etiology.

Objective: To present a topic review of the available scientific evidence regarding the impact of omentin-1, chemerin and miR-1013p gene expression in the development of GDM.

Materials and methods: A search of studies published during 2010-2019 about the three genes in women with GDM was carried out through MEDLINE and PUBMED.

Results: Eight articles met the inclusion criteria: four articles measured chemerin levels, three omentin-1 and one miR103p. It was shown that the circulation of chemerin and miR$103 p$ were positively correlated with the metabolic variables during the first and third trimester of pregnancy, it was also demonstrated that the omentin-1 release was higher in adipose tissue vs. placenta in cases of obesity.

Conclusion: Chemerin and miR-103p expression seems to related with the development of GDM.

Keywords: Diabetes, gestational, genes, obesity, adipose tissue, glucose.

\section{Introducción}

La diabetes mellitus gestacional (DMG) es una de las complicaciones obstétricas más comunes; esta es de origen multifactorial, relacionada con la predisposición genética, ganancia excesiva de peso, sedentarismo, hábitos alimentarios inadecuados y por trastornos del metabolismo de los hidratos de carbono (1, 2). La DMG se define como una intolerancia a los carbohidratos, caracterizada por disfunción de las células $\beta$ y resistencia a la insulina, que resulta en hiperglucemia de severidad variable de inicio durante el embarazo, considerándose el trastorno metabólico más frecuente durante la gestación $(3,4)$.

Se reconoce que, independientemente de las cifras de glucosa que se hayan alcanzado durante el embarazo, la mayoría de las mujeres con DMG regresan a parámetros normales des- 
pués del parto, sin embargo, estas presentan un mayor riesgo de desarrollar diabetes mellitus tipo 2 (DMT2), considerada una problemática de salud a nivel mundial, pues, de acuerdo con datos reportados por la Organización Mundial de Salud (OMS), la prevalencia ha aumentado del 4,7 \% en 1980 al 8,5 \% en el año 2014 y se ha proyectado que será la séptima causa de mortalidad en el año 2030 (5). Por otra parte, el Consenso de la Asociación Latinoamericana de Diabetes (ALAD) menciona que dos de cada cinco mujeres con diabetes se encuentran en edad reproductiva y la mitad del total de casos de hiperglucemia en el embarazo afecta a mujeres menores de 30 años, lo que representa más de 60 millones de mujeres en todo el mundo (6).

En México, la Encuesta Nacional de Salud y Nutrición (ENSANUT) 2016 indicó que el 10,3 \% de las mujeres reportan valores más elevados de glucosa que los hombres (8,4\%). Esta tendencia en México, por el solo hecho de ser mujer, agrega un mayor riesgo en la población mexicana femenina (10 \% en un porcentaje conservador) de desarrollar DMG que mujeres de otros países y, de hecho, si se agregan factores de riesgo, el porcentaje de las que pueden desarrollar esta complicación metabólica se eleva al $45 \%$ (7).

Dentro de los factores de riesgo para el desarrollo de DMG se consideran antecedentes de DMG en embarazos anteriores, DMT2 en familiares de primer grado, mortalidad perinatal, hijos macrosómicos, malformaciones congénitas, síndrome de ovario poliquístico (SOP), hipertensión arterial, glucosuria, edad $\geq 30$ años, índice de masa corporal (IMC) $\geq 27 \mathrm{~kg} / \mathrm{m}^{2}$ y obesidad $(6,8)$.

En lo que concierne al embarazo fisiológico, este se caracteriza por una serie de cambios endocrinos, metabólicos y vasculares, donde el almacenamiento de grasa materna aumenta. En este sentido, se ha comprobado que la DMG se asocia con un incremento de los niveles de ácidos grasos libres posprandiales debido a una capacidad reducida de insulina, que dificulta el proceso de lipólisis (9). Por ello, en la actualidad, la obesidad y la DMG constituyen una de las asociaciones más frecuentes y mortales para la madre, así como para su descendencia (10).

Recientemente, la investigación se ha centrado en el estudio de genes mediadores que podrían desempeñar un papel en el desarrollo de la DMG, como los derivados del tejido adiposo quemerina, omentina y miR-103p $(11,12)$. Estos genes tienen importantes funciones autocrinas/paracrinas, que regulan la diferenciación y proliferación celular, así como el metabolismo de lípidos, glucosa e intervienen en la apoptosis y biogénesis en el tejido adiposo (13-17).

\section{Quemerina (RARRES2 o TIG2)}

Es un gen que se expresa en el tejido adiposo de individuos con obesidad y actúa como un importante regulador de la inflamación, la aterosclerosis y la sensibilidad a la insulina (18). La unión de la quemerina con su receptor CMKLR ocasiona inhibición de la fosforilación del estimulador de la insulina y activación del adenosín monofosfato cíclico (AMPc) e indu- ce resistencia a la insulina en el músculo esquelético, siendo considerado uno de los principales genes del desarrollo de los componentes del síndrome metabólico (18).

En el 2014, van Poppel y colaboradores reportaron, en madres con DMG, que los niveles de quemerina en la sangre de la arteria fetal se elevaron en aproximadamente un $60 \%(p \leq 0,05)$ (19). Por otro lado, la quemerina de la sangre del cordón venoso fue mayor en los bebés de mujeres con obesidad $(p<0,01)$.

\section{Omentina-1 (ITLN1)}

La omentina-1 es un gen que se identificó por primera vez en 2003 a partir del tejido adiposo omental visceral. Hay dos isoformas altamente homólogas de omentina, omentina-1 y omentina-2; sin embargo, la omentina-1 es la principal forma circulante en el plasma humano, al relacionarse con la obesidad, DMT2, síndrome metabólico y SOP (20-22). La omentina tiene la capacidad de regular las actividades metabólicas relacionadas con la sensibilidad a la insulina y antiinflamatorias, lo que ofrece protección cardiovascular en las enfermedades como la obesidad y la DMT2 (22).

La omentina se sintetiza y secreta por diferentes tipos celulares, tales como preadipocitos, células madre mesenquimales, células progenitoras endoteliales, macrófagos, linfocitos B y linfocitos T (22). Esta hormona desempeña un papel importante en la modulación de la sensibilidad a la insulina mediante el factor paracrino y endocrino, donde aumenta la sensibilidad a la insulina y el metabolismo de la glucosa a nivel local del tejido adiposo omental. A medida que aumenta la transducción de la señal de la insulina a través de la activación, la proteína cinasa (Akt/mL proteína cinasa B) modula la distribución de la grasa corporal entre el depósito de grasa visceral y subcutánea (18). Por otro lado, ya que la omentina se secreta en la sangre humana, esta puede mejorar la sensibilidad a la insulina y el metabolismo de la glucosa en sitios distantes, como músculos, hígado y tejido adiposo subcutáneo (18-22). Con la información disponible hasta ahora, la expresión del gen de quemerina se ha considerado como un marcador de adiposidad (18).

Se han encontrado niveles más elevados de omentina en sujetos con peso saludable en comparación con sujetos con sobrepeso u obesos, independiente de la edad y el género; por otro lado, existe una correlación negativa entre los niveles plasmáticos de omentina y la medición de la resistencia a la insulina mediante el índice HOMA, IMC, circunferencia abdominal y niveles de leptina e insulina (22).

\section{MIR-103-3P}

Los miARN se han identificado como nuevos biomarcadores para el diagnóstico y pronóstico de diversas enfermedades, por ejemplo, los miARN derivados del tejido adiposo tienen un papel importante en la regulación de la expresión génica en el propio tejido adiposo (13). 
Entre los miARN implicados en la adipogénesis se encuentran miR-103, miR-146-b y miR-148-a, mientras que los miARN asociados con el desarrollo de la resistencia a la insulina son miR-103-3p, estos últimos afectan la vía de señalización del receptor de insulina $(8,23)$. En lo que concierne a miR-103 y miR-107, estos tienen un papel clave en la sensibilidad de la insulina, DMT2, obesidad y proliferación de células intestinales, debido a la inhibición de la traducción del ARNm o al potenciar la degradación del ARNm (23).

Jensen y colaboradores mencionan que los microARN están influenciados principalmente por factores no genético, en específico, los niveles de miR-103 se asocian con la adipogénesis del tejido adiposo subcutáneo, aumento del IMC y niveles de glucosa en plasma y hemoglobina glucosilada (HbA1c) en humanos (23).

El objetivo del presente trabajo fue presentar una revisión de tema de la evidencia científica disponible para describir el efecto de la expresión de los genes omentina-1, quemerina y miR-1013p, implicados en el desarrollo de la DMG.

\section{Materiales y métodos}

\section{Búsqueda de información}

Se realizó una búsqueda en las bases de datos MEDLINE y PUBMED en inglés y español, utilizando las palabras claves: (Chemerin/Omentin-1/miR103p[filter]) AND ("Diabetes, Gestational"[Mesh] OR “GDM") OR ("Diabetes, Pregnancy, Adipose Tissue" [Mesh].

\section{Estrategia de búsqueda}

Se hizo una revisión de los resultados de los genes y su relación con el desarrollo de la DMG. Este proceso de búsqueda tuvo tres etapas:

- Formulación del problema, donde se identificó el problema y se formuló la pregunta que guio la revisión de tema, así como el objetivo de esta.

- Localización y selección de estudios primarios, definiendo los criterios de selección de los estudios y las características de la población.

- Análisis y presentación de resultados, caracterizando los estudios.

La pregunta que guio la revisión de tema fue: ¿cuáles son las características de los resultados de los genes omentina-1, quemerina y miR-1013p en mujeres con DMG en las variables metabólicas de glucosa, insulina, Hb1Ac, HOMA-IR, con los niveles de quemerina, omentina-1 y miR103p?

\section{Selección de estudios}

Se incluyeron aquellos artículos de tipo transversales, descriptivos, publicados entre los años 2010 y 2019, siguiendo los criterios PRISMA. La población por estudiar fue mujeres cursando embarazos únicos con diagnóstico de DMG con mediciones de las adipocinas omentina-1, quemerina y miR-103p. Se excluyeron de la búsqueda de informa- ción los estudios de pacientes con diagnóstico de DMT1 o DMT2 (Figura 1).

\section{Extracción de datos}

Cada estudio se analizó de forma independiente por los autores. De esta manera se alcanzó un consenso, luego se agruparon para facilitar la comprensión de los resultados de todos los artículos revisados. El control de calidad de la información se llevó a cabo mediante tablas en las que se registró la siguiente información: nombre del autor, año, país escenario del estudio, nombre de la revista, factor de impacto y Qualis de la misma (Tabla 1).

\section{Resultados}

Siguiendo los criterios de búsqueda citados en el apartado anterior, a modo de búsqueda inicial se registraron 87 artículos. Así, se realizó una evaluación de esta primera muestra de artículos siguiendo los criterios comentados anteriormente, excluyéndose de los resultados de la presente revisión 80 artículos referentes a estudios no ajustados a la temática de la revisión, que son la expresión de los genes de omentina-1, quemerina y miR-103p. Así, al término del proceso de selección se escogieron ocho artículos, de los cuales, cuatro midieron los niveles de quemerina, tres de omentina- 1 y solo uno de miR103p (Figura 1) (24-26, 28-31).

Los artículos seleccionados procedieron de los siguientes países: Alemania, Australia, China, Corea, España, Grecia y el $100 \%$ de los artículos fue en inglés (24-26, 28-31). Este resultado puede estar relacionado con el hecho de que los estudios se encontraron en las bases de datos que abarcan revistas indexadas (Tabla 1). En relación con la distribución de las revistas de publicación de los estudios, se comprobó que el $100 \%$ es internacional. Se destaca el mayor factor de impacto atribuido al Journal of Clinical Endocrinology and Metabolism con 2,94 puntos según el Journal Citation Reports (JCR) (29). En cuanto a Qualis solo 5 revistas presentaron esa estratificación: 3-A1, 1-A2 y 1-B1 (Tabla 1) $(25,27,29,31)$.

La totalidad de los artículos revisados son estudios transversales (Tabla 2) (24-31). Siete estudios tuvieron lugar en hospitales de servicios especializados con tecnología intermedia, oferta de servicios médicos, de apoyo diagnóstico y terapéutico, atención de urgencia y emergencia (25-31). La media del número de muestra de pacientes que se utilizó en los estudios fue de 91, con un mínimo de 15 en el grupo control vs. 94 y un máximo de 144 participantes en el grupo control y 212 en el grupo a comparar (Tabla 2) $(27,29,30)$.

En el $57 \%$ de los estudios se explicaron los criterios de exclusión, mientras el 42,8 \% no explican los criterios de inclusión que guiaron sus estudios (24-30). Cabe mencionar que en la totalidad de los artículos no se explica cómo los autores realizaron el cálculo muestral (24-31). La mayoría de los estudios emplearon dos grupos para contrastar los resultados: 


\section{SISTEMA MINIMEDTM 670G EL PRIMER SISTEMA DE BOMBA DE INSULINA AUTOAJUSTABLE DEL MUNDO}

Se requiere cierta interacción del usuario.

$>$ Ajusta automáticamente la infusión de insulina basal, basándose en las lecturas de monitorización continua de glucosa (MCG).

$>$ La tecnología SmartGuard ${ }^{\mathrm{TM}}$ acerca el manejo de la diabetes a una tecnología totalmente automatizada.

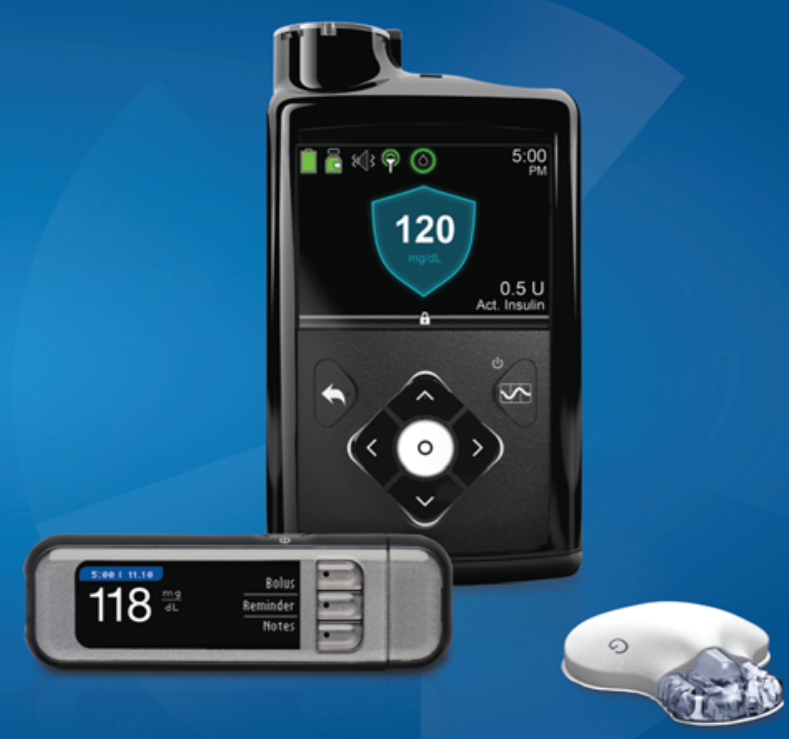

TERAPIA DE BOMBA TRADICIONAL

SIN AUTOMATIZACIÓN

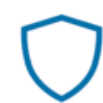
SMARTGUARDTM AJUSTES AUTOMÁTICOS DE INSULINA

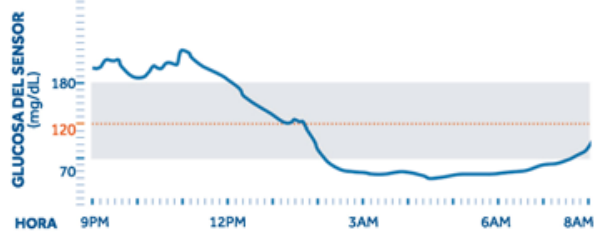

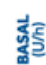

INFUSIÓN BASAL PROGRAMADA PREVIAMENTE

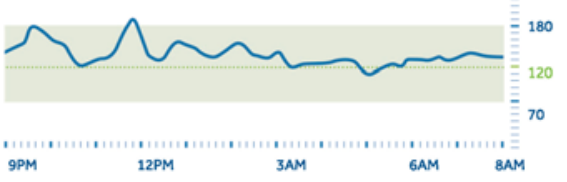

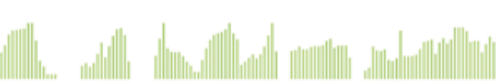

INFUSIÓN BASAL AJUSTADA AUTOMÁTICAMENTE

Ajusta la insulina basal automáticamente cada 5 minutos, basándose en los valores de glucosa del sensor para adaptarse a las necesidades únicas de insulina de los

pacientes.
Permite establecer múltiples patrones basales durante el dia y la noche, pero puede ser muy dificil predecir y adaptarse a las necesidades basales únicas que cambian diariamente.
RESULTADOS DELENSAYO PIVOTAL, ADULTOS (14-75 ANOS) $)^{2,3}$

\section{MODO AUTOMÁTICO DESMARTGUARD ${ }^{\text {TM }}$}

1

Reducción HbA1c:

$$
\underset{\text { visoncon }}{7,4} \rightarrow \underset{\text { v, }}{6,9}
$$

Incremento en Tiempo en Rango:

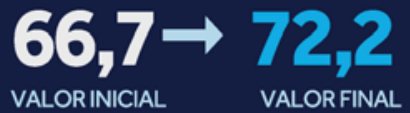

\section{SE ADAPTA A LAS NECESIDADES ÚNICAS DE CADA PACIENTE PARA AYUDAR A MAXIMIZAR SU TIEMPOENRANGO.}

CERO

CASOS DE HIPOGLUCEMIA GRAVE EN LAFASE DEL ESTUDIO

\section{cast}

Se requiere cierta interacción del usuario 
mujeres con embarazo sin DMG o con riesgo o diagnóstico de DMG (Tabla 3) (24, 27-29, 31).

Cabe destacar que los criterios de diagnóstico de DMG utilizados en los estudios fueron a través del examen de tolerancia oral a la glucosa (27-34). Esta prueba se emplea para la detección de diabetes tipo 2 (33). El análisis de la expresión de los genes de quemerina y omentina-1 se llevó a cabo a través del ensayo inmunoabsorbente ligado a enzimas (ELISA), mientras que la medición de la expresión de miR-103p fue con extracción y purificación de miARN (Tabla 3) (24-31). Con respecto a los datos obtenidos en los estudios se establecieron tres apartados:

\section{Quemerina y DMG}

En 2010, Pfau y colaboradores realizaron un estudio transversal para investigar los niveles de quemerina en la circulación en 40 mujeres con DMG vs. 80 pacientes sin DMG, emparejadas por la edad gestacional e insulina en ayunas (24). Se encontró que los niveles séricos de quemerina se correlacio-

Tabla 1. Caracterización de las revistas de los estudios analizados

\begin{tabular}{|c|c|c|c|c|c|}
\hline & Autor, año & $\begin{array}{c}\text { País escenario } \\
\text { del estudio }\end{array}$ & Nombre de la revista & $\begin{array}{l}\text { Factor de impacto } \\
\text { de la revista }\end{array}$ & Qualis \\
\hline \multirow[t]{4}{*}{ Quemerina } & Pfau y colaboradores, 2010 (24) & Alemania & Homem Research in Pediatrics & 1,7 & - \\
\hline & Ye y colaboradores 2017 (25) & China & Gynecological Endocrinology & 1,04 & B1 \\
\hline & Cheon y colaboradores 2017 (26) & Corea & Yonsei Medical Journal & 1,564 & - \\
\hline & $\begin{array}{l}\text { Panayoula y colaboradores, } \\
2018 \text { (27) }\end{array}$ & Grecia & Peptides & 2,851 & A2 \\
\hline \multirow[t]{3}{*}{ Omentina-1 } & Barker y colaboradores, 2012 (28) & España & Plos One & 1,16 & A1 \\
\hline & $\begin{array}{l}\text { Abell y colaboradores, } \\
2017(29)\end{array}$ & Australia & $\begin{array}{c}\text { The Journal of Clinical Endocri- } \\
\text { nology and Metabolism }\end{array}$ & 2,94 & A1 \\
\hline & $\begin{array}{l}\text { Abell y colaboradores, } \\
\qquad 2018(30)\end{array}$ & Australia & $\begin{array}{c}\text { The Australian and New Zea- } \\
\text { land Journal of Obstetrics and } \\
\text { Gynecology (ANZJOG) }\end{array}$ & 1,766 & - \\
\hline miARN-103p & $\begin{array}{l}\text { Carreras-Badosa y } \\
\text { colaboradores, } 2015(31)\end{array}$ & España & $\begin{array}{c}\text { Journal Clinical Endocrinology } \\
\text { and Metabolism }\end{array}$ & 2,842 & A1 \\
\hline
\end{tabular}

Figura 1. Flujograma de información de las etapas de búsqueda y selección de estudios bajo la declaración PRISMA (32).

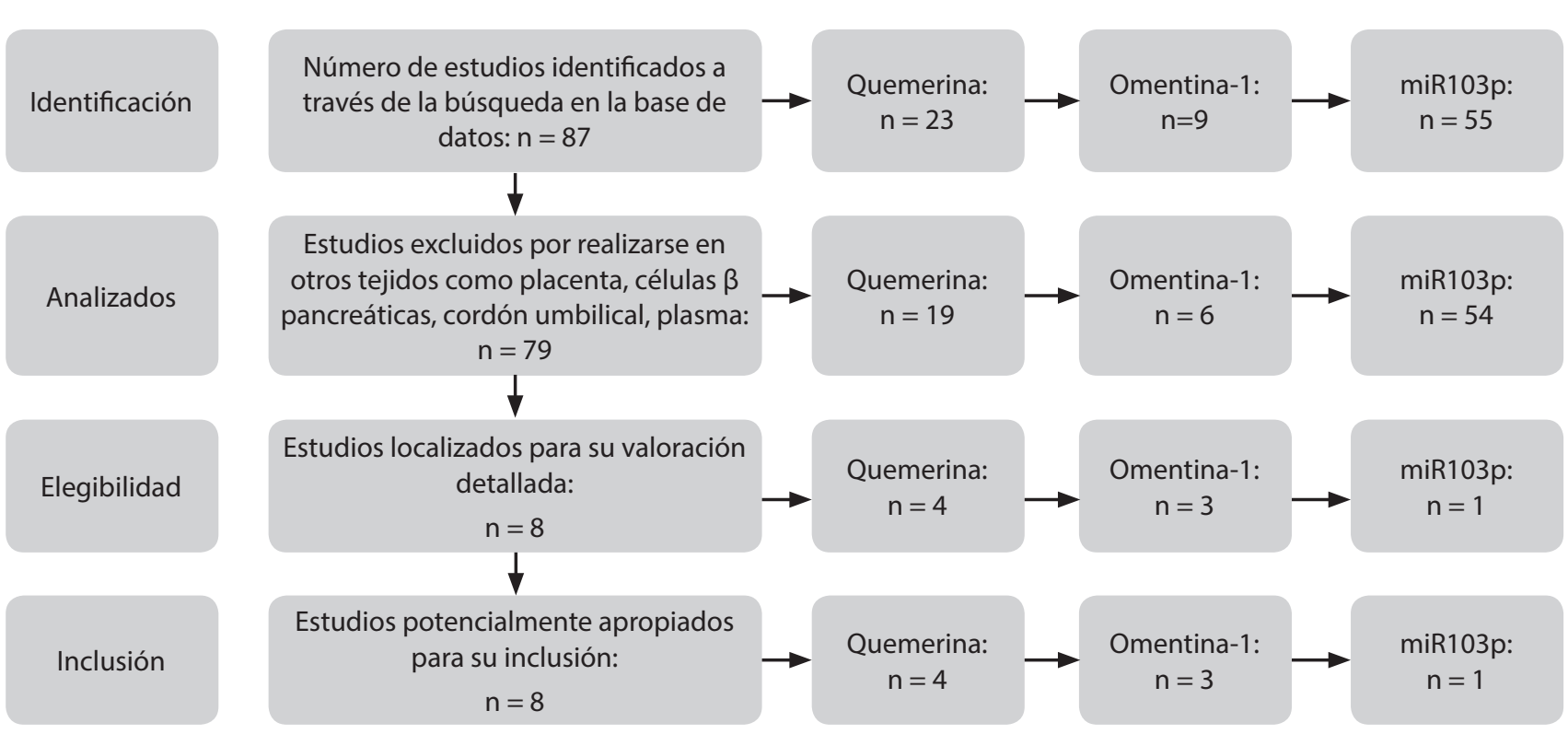


Tabla 2. Caracterización de los estudios analizados

\begin{tabular}{|c|c|c|c|c|}
\hline & $\begin{array}{l}\text { Autor, año, país } \\
\text { escenario del } \\
\text { estudio }\end{array}$ & $\begin{array}{l}\text { Tipo de } \\
\text { estudio }\end{array}$ & $\mathbf{N}$ & Objetivo \\
\hline \multirow[t]{4}{*}{ Quemerina } & $\begin{array}{l}\text { Pfau y } \\
\text { colaboradores, } \\
\text { 2010, Alemania (24) }\end{array}$ & Transversal & $\begin{array}{l}\text { Pacientes de control } \\
(n=80) \text { y DMG }(n=40)\end{array}$ & $\begin{array}{l}\text { Investigar los niveles de quemerina en } \\
\text { circulación en pacientes con DMG vs. } \\
\text { pacientes sin DMG emparejadas por la } \\
\text { edad gestacional e insulina en ayunas }\end{array}$ \\
\hline & $\begin{array}{l}\text { Ye y colaboradores, } \\
2017, \text { China }(25)\end{array}$ & Transversal & $\begin{array}{l}212 \text { mujeres a las 8-12 SDG. Después de } \\
\text { la detección de DMG se incluyeron } 19 \\
\text { con DMG y } 20 \text { al azar de } 144 \text { mujeres } \\
\text { con TNG que acudieron al Hospital } \\
\text { Central Xiangyang }\end{array}$ & $\begin{array}{l}\text { Investigar la asociación entre el nivel de } \\
\text { quemerina en el primer trimestre del } \\
\text { embarazo y el riesgo de DMG }\end{array}$ \\
\hline & $\begin{array}{l}\text { Cheon y } \\
\text { colaboradores, } \\
2017, \text { Corea }(26)\end{array}$ & Transversal & $\begin{array}{l}102 \text { mujeres recién diagnosticadas con } \\
\text { DMG que acudieron al departamen- } \\
\text { to ambulatorio de Endocrinología y } \\
\text { Metabolismo en el Hospital Sagrado } \\
\text { Corazón de Hallym University en el } \\
\text { período de mayo } 2009 \text { a mayo } 2010\end{array}$ & $\begin{array}{l}\text { Investigar la relación entre los niveles } \\
\text { séricos de quemerina y TA visceral }\end{array}$ \\
\hline & $\begin{array}{l}\text { Panayoula y } \\
\text { colaboradores, } \\
\text { 2018, Grecia (27) }\end{array}$ & Transversal & $\begin{array}{l}\text { De } 38 \text { mujeres embarazadas para } \\
\text { el fin de los estudios: } 23 \text { mujeres } \\
\text { embarazadas (TNG) y } 15 \text { con DMG } \\
\text { que acudieron al Hospital Central } \\
\text { Universitario }\end{array}$ & $\begin{array}{l}\text { Examinar si las adipocinas circulantes } \\
\text { y su expresión de ARNm en el tejido } \\
\text { adiposo y la placenta están alteradas } \\
\text { en el embarazo con DMG }\end{array}$ \\
\hline \multirow[t]{3}{*}{ Omentina-1 } & $\begin{array}{l}\text { Barker y } \\
\text { colaboradores, } \\
\text { 2012, España (28) }\end{array}$ & Transversal & $\begin{array}{l}44 \text { mujeres con TNG y con obesidad } \\
\text { y } 39 \text { mujeres sin obesidad con DMG } \\
(\mathrm{n}=39 \text { ) que acudieron al Hospital de } \\
\text { la Misericordia para la investigación } \\
\text { de la mujer }\end{array}$ & $\begin{array}{l}\text { Determinar el efecto de la obesidad } \\
\text { materna y DMG sobre los niveles circu- } \\
\text { lantes de omentina-1 en el cordón um- } \\
\text { bilical y el plasma materno, expresión } \\
\text { de genes y liberación de omentina-1 de } \\
\text { la placenta humana y del TA }\end{array}$ \\
\hline & $\begin{array}{l}\text { Abell y } \\
\text { colaboradores, } \\
\text { 2017, Australia (29) }\end{array}$ & Transversal & $\begin{array}{l}103 \text { mujeres con embarazo nor- } \\
\text { moevolutivo y factores de riesgo } \\
\text { a desarrollar DMG que acudieron } \\
\text { al Hospital de Salud Monash en } \\
\text { Melbourne }\end{array}$ & $\begin{array}{l}\text { Investigar la asociación de adipocinas } \\
\text { y otras sustancias inflamatorias con } \\
\text { desarrollo de DMG utilizando muestras } \\
\text { de suero de ECA }\end{array}$ \\
\hline & $\begin{array}{l}\text { Abell y } \\
\text { colaboradores, } \\
\text { 2018, Australia (30) }\end{array}$ & Transversal & $\begin{array}{l}94 \text { mujeres control } \\
40 \text { mujeres con DMG }\end{array}$ & $\begin{array}{l}\text { Prevenir el exceso de peso durante } \\
\text { el embarazo en mujeres en riesgo de } \\
\text { DMG }\end{array}$ \\
\hline miARN-103p & $\begin{array}{l}\text { Carreras-Badosa } \\
\text { y colaboradores, } \\
2015, \text { España }(31)\end{array}$ & Transversal & $\begin{array}{l}70 \text { mujeres embarazadas que acu- } \\
\text { dieron al Hospital Dr. Josep Trueta }\end{array}$ & $\begin{array}{l}\text { Definir el patrón de los miARN circu- } \\
\text { lantes en la obesidad gestacional y } \\
\text { pregestacional y explorar sus asocia- } \\
\text { ciones con los parámetros metabólicos } \\
\text { maternos y con marcadores para el } \\
\text { crecimiento pre y posnatal }\end{array}$ \\
\hline
\end{tabular}

DMG: diabetes mellitus gestacional, ECA: ensayo controlado aleatorio, IMC: índice de masa corporal, TA: tejido adiposo, TNG: tolerancia normal a la glucosa.

naron positivamente con la presión arterial sistólica, presión arterial diastólica, creatinina sérica, insulina en ayunas, HOMA-IR, triglicéridos y leptina $(p<0,05)$. Las mujeres con DMG tuvieron valores de glucosa más altos en comparación con los controles $(p=0,001)$ (24).

En 2017, Ye y colaboradores realizaron un estudio transversal para investigar la asociación entre el nivel de quemeri- na en el primer trimestre del embarazo y el riesgo de DMG en 212 mujeres, con una edad gestacional de 8-12 semanas (SDG) (25). Después de la detección de DMG se incluyeron 19 pacientes con DMG y 20 al azar en 144 mujeres con tolerancia normal a la glucosa (TNG). Los autores reportaron que los niveles de quemerina en el grupo DMG $(146,60 \pm 38,91 \mathrm{ng} / \mathrm{mL})$ fueron significativamente más bajos vs. el grupo TNG $(187,23 \pm 46,83$ 
Tabla 3. Características de diseño de los estudios incluidos

\begin{tabular}{|c|c|c|c|c|c|c|}
\hline & $\begin{array}{l}\text { Autor, año, } \\
\text { país escenario } \\
\text { del estudio }\end{array}$ & Grupos & $\begin{array}{l}\text { Criterios de } \\
\text { selección }\end{array}$ & $\begin{array}{l}\text { Cálculo de } \\
\text { tamaño } \\
\text { muestral }\end{array}$ & $\begin{array}{c}\text { Criterios de } \\
\text { diagnóstico de } \\
\text { DMG }\end{array}$ & $\begin{array}{c}\text { Técnica de medición de } \\
\text { genes }\end{array}$ \\
\hline \multirow[t]{4}{*}{ Quemerina } & $\begin{array}{l}\text { Pfau y } \\
\text { colaboradores, } \\
\text { 2010, Alemania } \\
(24)\end{array}$ & $\begin{array}{l}\text { Grupo control } \\
\text { DMG }\end{array}$ & $\begin{array}{l}\text { Inclusión: no } \\
\text { Exclusión: sí }\end{array}$ & No & $\begin{array}{l}\text { PTGO } 75 \mathrm{~g}: \\
\text { Ayuno: } 5,3 \mathrm{mmol} / \mathrm{L} \\
1 \mathrm{h:} 10,0 \mathrm{mmol} / \mathrm{L} \\
2 \mathrm{h:} 8,6 \mathrm{mmol} / \mathrm{L}\end{array}$ & $\begin{array}{l}\text { Quemerina determinada } \\
\text { con ELISA de acuerdo con } \\
\text { las instrucciones de los } \\
\text { fabricantes (BioVendor, Mo- } \\
\text { drice, República Checa) }\end{array}$ \\
\hline & $\begin{array}{l}\text { Ye y } \\
\text { colaboradores, } \\
\text { 2017, China } \\
(25)\end{array}$ & $\begin{array}{l}\text { Mujeres con } \\
\text { TNG } \\
\text { DMG }\end{array}$ & $\begin{array}{l}\text { Inclusión: no } \\
\text { Exclusión: sí }\end{array}$ & No & $\begin{array}{l}\text { PTGO } 75 \mathrm{~g}: \\
\text { criterios de IADPSG: } \\
\text { Ayuno: } 5,1 \mathrm{mmol} / \mathrm{L} \\
1 \mathrm{~h}: 10,0 \mathrm{mmol} / \mathrm{L} \\
2 \mathrm{~h}: 8,5 \mathrm{mmol} / \mathrm{L})\end{array}$ & $\begin{array}{l}\text { Las concentraciones séricas } \\
\text { de quemerina se midieron } \\
\text { utilizando kits ELISA Min- } \\
\text { neapolis, MN, de acuerdo } \\
\text { con las instrucciones del } \\
\text { fabricante }\end{array}$ \\
\hline & $\begin{array}{l}\text { Cheon y } \\
\text { colaboradores, } \\
2017, \text { Corea } \\
(26) \\
\end{array}$ & $\begin{array}{l}\text { Grupo control } \\
\text { DMG }\end{array}$ & $\begin{array}{l}\text { Inclusión: sí } \\
\text { Exclusión: sí }\end{array}$ & No & $\mathrm{HbA1c}$ & $\begin{array}{l}\text { Los niveles de quemerina } \\
\text { se midieron utilizando un } \\
\text { kit de inmunoensayo enzi- } \\
\text { mático (Mesdia, Seúl, Corea }\end{array}$ \\
\hline & $\begin{array}{l}\text { Panayoula y } \\
\text { colaboradores, } \\
2018, \text { Grecia } \\
(27)\end{array}$ & $\begin{array}{l}\text { Mujeres } \\
\text { embarazadas } \\
\text { con TNG y } \\
\text { mujeres con } \\
\text { DMG }\end{array}$ & $\begin{array}{l}\text { Inclusión: sí } \\
\text { Exclusión: sí }\end{array}$ & No & $\begin{array}{l}\text { PTGO } 75 \mathrm{~g}: \\
\text { Ayuno: } 95 \mathrm{mg} / \mathrm{dL} \\
1 \mathrm{~h}: 180 \mathrm{mg} / \mathrm{dL} \\
2 \mathrm{h:} 155 \mathrm{mg} / \mathrm{dL}\end{array}$ & $\begin{array}{l}\text { La cuantificación de leo } \\
\text { genes se realizó por medio } \\
\text { de PCR (LightCycler, ROCHE, } \\
\text { Manheim, Germany) }\end{array}$ \\
\hline \multirow[t]{3}{*}{ Omentina-1 } & $\begin{array}{l}\text { Barker y } \\
\text { colaboradores, } \\
\text { 2012, España } \\
(28)\end{array}$ & $\begin{array}{l}\text { Grupo control } \\
\text { DMG }\end{array}$ & $\begin{array}{l}\text { Inclusión: sí } \\
\text { Exclusión: no }\end{array}$ & No & $\begin{array}{l}\text { PTGO } 75 \mathrm{~g}: \\
\text { ADIPS } \\
\text { Ayuno: } 5,5 \mathrm{mmol} / \mathrm{L} \\
2 \mathrm{h:} 8,0 \mathrm{mmol} / \mathrm{L}\end{array}$ & $\begin{array}{l}\text { El kit ELISA omentina-1 se } \\
\text { obtuvo de Cusabio Biotech } \\
\text { Co. y se realizó de acuerdo } \\
\text { con las instrucciones de los } \\
\text { fabricantes }\end{array}$ \\
\hline & $\begin{array}{l}\text { Abell y } \\
\text { colaboradores, } \\
\text { 2017, Australia } \\
(29)\end{array}$ & $\begin{array}{l}\text { Grupo control } \\
\text { con riesgo de } \\
\text { DMG }\end{array}$ & $\begin{array}{l}\text { Inclusión: sí } \\
\text { Exclusión: no }\end{array}$ & No & $\begin{array}{l}\text { Criterios de } \\
\text { diabetes en el } \\
\text { embarazo Australia } \\
\text { (ADIPS 1998) } \\
\text { Ayuno: } 5,5 \mathrm{mmol} / \mathrm{L} \\
2 \mathrm{~h}: 8,0 \mathrm{mmol} / \mathrm{L}\end{array}$ & $\begin{array}{l}\text { Omentina-1 se midió } \\
\text { utilizando el ELISA Millipore } \\
\text { (EZHOMNTN1-29K) }\end{array}$ \\
\hline & $\begin{array}{l}\text { Abell y } \\
\text { colaboradores, } \\
2018, \text { Australia } \\
(30)\end{array}$ & $\begin{array}{l}\text { Grupo control } \\
\text { mujeres con } \\
\text { DMG }\end{array}$ & $\begin{array}{l}\text { Inclusión: sí } \\
\text { Exclusión: no }\end{array}$ & No & $\begin{array}{l}\text { Criterios de diabe- } \\
\text { tes en el embarazo } \\
\text { Australia (ADIPS } \\
\text { 1998) } \\
\text { Ayuno: } 5,5 \mathrm{mmol} / \mathrm{L} \\
2 \mathrm{~h}: 8,0 \mathrm{mmol} / \mathrm{L}\end{array}$ & $\begin{array}{l}\text { Omentina-1 se midió } \\
\text { utilizando el ELISA Millipore } \\
\text { (EZH0MNTN1-29K) }\end{array}$ \\
\hline miARN-103p & $\begin{array}{l}\text { Carreras- } \\
\text { Badosa y } \\
\text { colaboradores, } \\
\text { 2015, España } \\
\text { (31) }\end{array}$ & $\begin{array}{l}\text { Mujeres emba- } \\
\text { razadas } \\
\text { Control sin } \\
\text { obesidad } \\
\text { Embarazo más } \\
\text { obesidad } \\
\text { Embarazo más } \\
\text { peso pregesta- } \\
\text { cional }\end{array}$ & $\begin{array}{l}\text { Inclusión: sí } \\
\text { Exclusión: no }\end{array}$ & No & & $\begin{array}{l}\text { La extracción y purificación } \\
\text { de miARN circulante se obtu- } \\
\text { vo mediante la venopunción } \\
\text { estándar y la centrifugación } \\
\text { EDTA-coated Vacutainer } \\
\text { Tubes (BectonDickinson). } \\
\text { La cantidad de ARN aislado } \\
\text { se determinó con aNano- } \\
\text { dropND-1000 espectrofotó- } \\
\text { metro (Nanodrop Techno- } \\
\text { logies) }\end{array}$ \\
\hline
\end{tabular}

ADIPS: Australasian Diabetes in Pregnancy Society; DMG: diabetes mellitus gestacional; IADPSG: Asociación Internacional de Grupos de Estudios sobre Diabetes y Embarazo; PTGO: prueba de tolerancia oral a la glucosa; TNG: tolerancia normal a la glucosa. 
$\mathrm{ng} / \mathrm{mL})$ durante el primer trimestre $(p<0,05)$, pero más alto durante el tercer trimestre del embarazo en el grupo de DMG vs. TNG (308,56 $\pm 56,43 \mathrm{ng} / \mathrm{mL} v$ s. 227,53 $\pm 46,49 \mathrm{ng} / \mathrm{mL}, p<0,05)$, correlacionándose positivamente con la aparición de DMG durante el primer trimestre $(p=0,05)$. El riesgo de DMG se asoció positivamente con el nivel sérico de quemerina en el primer trimestre ( $\mathrm{r} 2=0,652, p=0,001)$, ajustado por IMC (25).

En 2017, Cheon y colaboradores realizaron un estudio transversal en 102 mujeres recién diagnosticadas con DMG para investigar la relación entre los niveles séricos de quemerina y la adiposidad visceral en el período de mayo 2009 a mayo 2010 (26). Se reportó que los niveles séricos de quemerina mostraron correlaciones positivas significativas con IMC, circunferencia de cintura y grasa visceral, así como con los niveles de HOMA-IR y niveles de triglicéridos (26).

En 2018, Panayoula y colaboradores realizaron un estudio transversal en 38 mujeres embarazadas ( 23 con TNG y 15 con DMG) y encontraron que la expresión del ARNm de quemerina en tejido adiposo subcutáneo y visceral fue significativamente mayor en comparación con la expresión en placenta ( 8 a 24 veces, $p$ $<0,02$ y 6 a 10 veces $p<0,05$, respectivamente) (27). Más aún, en casos de obesidad con DMG, la expresión de ARNm de quemerina en tejido visceral fue significativamente mayor comparada con la que se registró de mujeres con TNG sin obesidad (16,12 \pm 9,16 vs. 5,51 $\pm 1,41, p<0,05$ ), la cual se mantuvo significativa después del ajuste por edad y HOMA-IR [F $(1,19)=5,91, p=0,025]$ (27).

\section{Omentina y DMG}

En 2012, Barker y colaboradores realizaron un estudio para determinar el efecto de la obesidad materna y DMG sobre los niveles circulantes de omentina-1 en el cordón umbilical y el plasma materno, y la expresión de genes y la liberación de omentina-1 de la placenta humana y tejido adiposo en $44 \mathrm{mu}$ jeres con TNG con obesidad y 39 con DMG sin obesidad (28). Se comprobó que los niveles de omentina-1 fueron mayores de las 11 SDG en comparación con las 28 SDG. Agregándose a este dato también se encontró que la liberación de omentina-1 fue mayor en la placenta en comparación con el tejido adiposo. Sin embargo, la expresión génica de omentina-1 fue significativamente inferior en placenta y tejido adiposo obtenido de mujeres con obesidad materna preexistente (28).

En 2017, Abell y colaboradores realizaron un estudio transversal para investigar la asociación de adipocinas y otras sustancias inflamatorias con el desarrollo de DMG en 103 mujeres con embarazo normoevolutivo y factores de riesgo para desarrollar DMG (29). En su abordaje encontraron que la obesidad materna se asoció con niveles significativamente más bajos de omentina-1 en el plasma materno y que la expresión del gen omentina- 1 fue menor en la placenta y en el tejido adiposo obtenido de mujeres con obesidad preexistente. Además de esto, la liberación de omentina-1 en el tejido adiposo fue significativamente menor en las embarazadas con obesidad (29).
En 2018, el mismo grupo de Abell y colaboradores realizó un estudio transversal para prevenir la excesiva ganancia de peso en mujeres con riesgo de desarrollar DMG en 94 mujeres control y en 40 mujeres con DMG, sin obtener asociación entre los niveles de omentina-1 e IL-6 con biomarcadores de riesgo de desarrollar DMG de acuerdo con la sensibilidad y la especificidad de la prueba del área bajo la curva y las características maternas (30).

\section{miR103p y DMG}

En 2015, Carreras-Badosa y colaboradores condujeron un estudio transversal para definir el patrón de los miARN circulantes en la obesidad gestacional y pregestacional y explorar sus asociaciones con los parámetros metabólicos maternos y con marcadores para el crecimiento pre y posnatal en 70 mujeres embarazadas, en las que hallaron que 13 de los 18 miARN se encontraban presentes de manera diferente entre mujeres con embarazos normales y mujeres con DMG (31). Entre ellos se demostró que miR103p está asociado con aumento de peso durante el embarazo y se encontró una asociación positiva con las vías reguladoras de señalización de la insulina, considerándose, por tanto, un importante regulador de la adipogénesis (31).

\section{Discusión}

La DMG surge cuando se presenta resistencia a la insulina y reducción en la secreción de la insulina. Estos dos elementos pueden verse afectados por la cantidad de tejido adiposo pregestacional y su aumento durante la gestación. Como consecuencia, un papel fisiopatológico importante podría estar cubierto por expresión de genes que directa o indirectamente, a través de una respuesta inflamatoria crónica, agravaría la resistencia a la insulina, lo que promueve el inicio de DMG (34).

Está bien establecido que el tejido adiposo tiene un papel más allá de la regulación energética y que la expresión de genes tiene un papel importante en el desarrollo de complicaciones relacionadas con la obesidad (23). La evaluación simultánea de la expresión de diferentes genes a partir del tejido adiposo y las correlaciones con los parámetros clínicos es importante para comprender mejor la aparición de la DMG. En este trabajo de revisión de tema se analizaron ocho artículos, de los cuales, cuatro midieron los niveles de quemerina, tres de omentina-1y solo uno de miR-103p (24-31).

De acuerdo con los datos obtenidos, los niveles de quemerina mostraron una fuerte asociación con las cifras de presión arterial, insulina, HOMA-IR, triglicéridos y leptina, así como con la función renal, en comparación con las embarazadas sanas (24). En el estudio de Cheon se confirma que los niveles de quemerina se han implicado en la diferenciación de adipocitos y la estimulación de la lipólisis; además, esta hormona disminuye la absorción de glucosa basal e induce la resistencia a la insulina (26). Con base en los resultados de esta revisión se puede afirmar que en mujeres con DMG, los niveles de queme- 
rina fueron mayores en el tercer trimestre en comparación con el primer trimestre (25). Los resultados son consistentes con los informes anteriores, que han confirmado que los niveles séricos de quemerina están relacionados con la edad gestacional (32-35).

También se observó que las concentraciones de quemerina mostraron una correlación positiva con el IMC, circunferencia de cintura, grasa visceral, HOMA-IR y niveles de triglicéridos (27). Estos resultados confirman los hallazgos que mencionan que la quemerina se asocia significativamente con el IMC, circunferencia de cintura, así como con diversos componentes del síndrome metabólico (36).

La información encontrada indica que la expresión de quemerina en el tejido adiposo subcutáneo y visceral es significativamente mayor en comparación con la expresión en placenta (27). Al respecto se sabe que la cantidad de tejido adiposo visceral es una medida predictora importante de riesgo cardiometabólico, sin embargo, se ha sugerido que la razón entre los componentes visceral y subcutáneo puede ser un indicador más apropiado para evaluar la predisposición para acumular grasa visceral y las alteraciones cardiometabólicas, como la diabetes (37). Por otra parte, en 2010, Bozaoglu y colaboradores mencionaron que los niveles plasmáticos de quemerina son hereditarios y tienen un papel como estimulador de la angiogénesis y hacen hincapié en el hecho de que los niveles de quemerina en plasma son mayores en las mujeres que en los hombres, además, aumentan con la edad y con los componentes del síndrome metabólico (38).

Los estudios que midieron la expresión del gen de omentina-1 hallaron cifras mayores en placenta en comparación con el tejido adiposo (28-30). Sin embargo, cuando se presenta obesidad en la mujer, estas cifras disminuyen significativamente (28). A la omentina se le ha atribuido la capacidad de regular la sensibilidad a la insulina, lo que ofrece protección cardiovascular en casos de diabetes (20). Esto concuerda con lo expuesto por Tan y colaboradores, en 2008, quienes hallaron una disminución de omentina-1 y aumento de las variables de insulina y glucosa en mujeres con SOP en comparación con un grupo control (36).

Una limitación de este estudio es que los criterios de calidad para seleccionar los artículos podrían parecer laxos; sin embargo, debido a la escasez de resultados con los genes de interés y el tema de diabetes gestacional, habríamos obtenido información extremadamente escasa.

En su conjunto, la información hallada revela que la omentina-1 está regulada por la insulina y la glucosa; esto puede, en parte, explicar la disminución de los niveles de omentina-1observada en mujeres con sobrepeso (7). Sin embargo, también hay algunas discrepancias, pues no se encontraron asociaciones entre la omentina-1 e IL-6 con biomarcadores de riesgo para el desarrollo de DMG (35). Finalmente, los estudios con el análisis de la expresión de miR-103p en mujeres embarazadas reportan una disminución de este gen en los casos metabólicos más adversos; es decir, mayor incremento de peso durante el embarazo, así como de los niveles de glucosa, HbA1c, HOMA-IR y triglicéridos (31). En los embarazos con obesidad, los miARN derivados del tejido adiposo pueden comunicarse con la placenta e inducir cambios en su función, que pueden contribuir al desarrollo de la DMG (39).

\section{Conclusión}

La expresión de genes en el acúmulo excesivo de tejido adiposo en la mujer gestante contribuye con el desarrollo de la DMG y de las complicaciones en la madre y su descendencia. Se recomienda aumentar la investigación para poder extraer datos más concluyentes sobre los genes y la relación con el metabolismo de la glucosa. El análisis bibliográfico también permitió identificar que se carece de publicaciones en revistas en español.

Los estudios se concentran en artículos de tipo transversal y enfoque cuantitativo. Sin embargo, la escasez de estudios experimentales muestra la necesidad de realizar estudios clínicos prospectivos. La evaluación de riesgo de DMG se debe realizar durante la primera visita prenatal en mujeres con factores de riego. La Asociación Internacional de Grupos de Estudio de Diabetes y Embarazo (IADPSG), utilizada en los artículos como criterios para diagnosticar la DMG, es una herramienta de detección masiva para la DMG $(25,28-30,40)$.

En el 2010, la IADPSG estableció los nuevos criterios diagnósticos para DMG, adoptada en 2013 por la OMS y en 2014 por la Asociación Americana de Diabetes (ADA), que se basa en el riesgo de resultados adversos durante el embarazo, donde un solo valor alterado es suficiente para establecer el diagnóstico (40). Con estos criterios diagnósticos, la prevalencia de diabetes gestacional se eleva aproximadamente $18 \%$, con repercusión en los costos de los sistemas de salud. Sin embargo, se sugiere que la prueba oral a la glucosa se realice cuando se presentan factores de riesgo, para su mejor diagnóstico y tratamiento.

Con respecto al tipo de estudio, el $100 \%$ corresponde al tipo transversal. Este tipo de diseño se caracteriza por una única medida de las variables de interés, no habiendo seguimiento ni control en la fase de diseño de las variables predictoras (24-31, 39). Dado que la exposición y el resultado son examinados al mismo tiempo, existe una ambigüedad temporal en su relación, lo que impide establecer inferencias causa-efecto (41).

En cuanto al análisis del sistema Qualis, solo cinco estudios analizados de las revistas indexadas a las que pertenecen son sometidos a la clasificación Qualis, la cual se actualiza anualmente y sigue una serie de criterios definidos por la Coordinación de Perfeccionamiento de Personal de Enseñanza Superior (CAPES), tales como número de temas, indexación, número de instituciones editoriales, factor de impacto basado en JCR. Las calificaciones, llamadas estratos, ocurren en una escala (A1, la más alta; A2; B1; B2; B3; B4; B5; C; con puntaje cero) $(28,30-35,42)$. 


\section{DIGNÓSTICO DE HFHO NO HAY TIEMPO QUE PERDER}

12.5

años de edad

Edad a la cual son

alcanzados los límites

del riesgo

cardiovascular²

es potencialmente mortal si no es tratada. En esta rara enfermedad genética, el colesterol LDL se acumula rápidamente desde el nacimiento. ${ }^{1}$

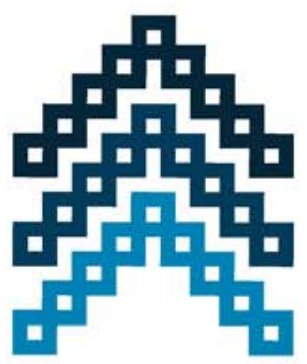

Aumento del riesgo de mortalidad

- Alterosclerosis severa y acelerada

- Enfermedad cardiovascular

- Muerte prematura

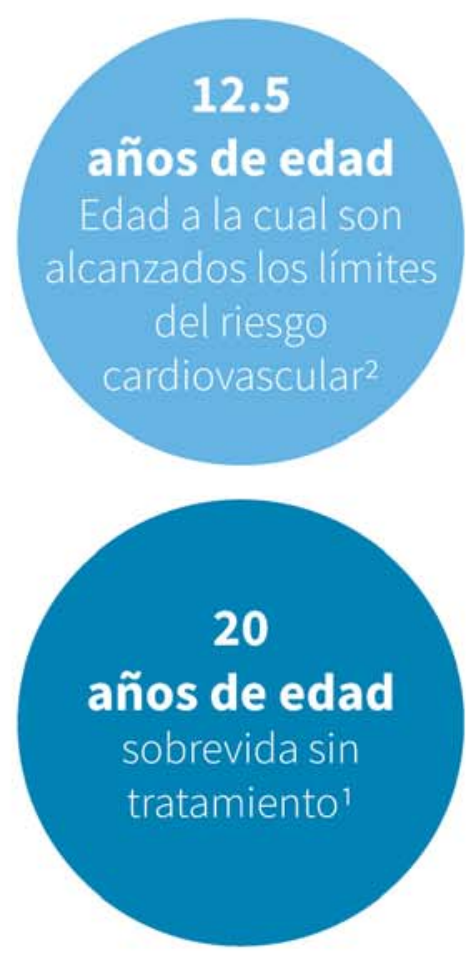

\section{SIN TRATAMIENTO EL RIESGO CARDIOVASCULAR INCREMENTA CADA AÑO ${ }^{2,4}$}

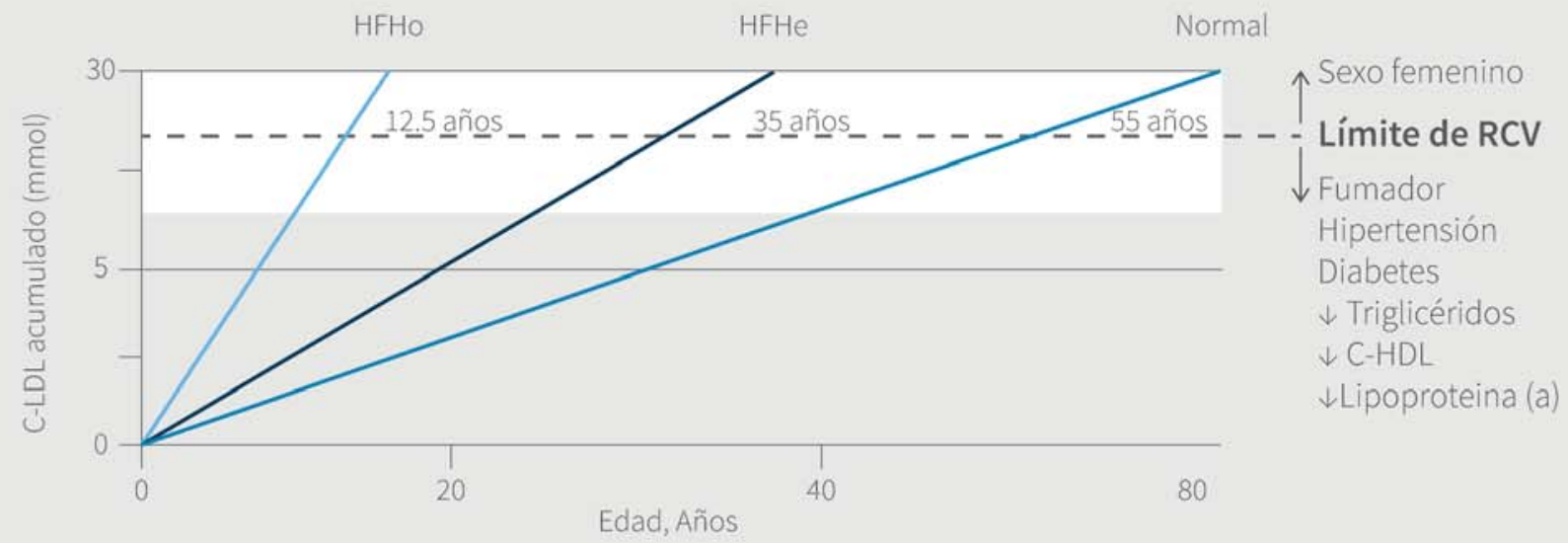

Figura: Efecto de acumulación de colesterol LDL en el riesgo cardiovascular según la edad 3,4

En la HFHo, la inabilidad de depurar los niveles de LDL resulta con una rápida acumulación. Un niño con 12.5 años de edad y con diagnóstico de HFHo tiene la misma carga de colesterol y niveles de asterosclerosis que una persona de 55 a 65 años $\sin \mathrm{HFHO}^{2,4}$ 


\section{Conflictos de interés}

Todos los autores declaran no tener conflictos de interés de ninguna índole.

\section{Fuentes de financiación}

El presente estudio no recibió patrocinio de ningún tipo.

\section{Agradecimientos}

Los autores desean agradecer a las instituciones que han hecho posible la realización del trabajo presentado, al Consejo Nacional de Ciencia y Tecnología, al Hospital Materno Perinatal “Mónica Pretelini Sáenz" y a la Universidad Autónoma del Estado de México.

\section{Referencias}

1. Flores L, Solorio I, Melo M, Trejo J. Embarazo y obesidad: riesgo para desarrollo de diabetes gestacional en la frontera norte de México. Gac Méd Méx. 2014;150,73-8.

2. Farias J, Pérez C, Saavedra D. Diabetes mellitus gestacional: una aproximación a los conceptos actuales sobre estrategias diagnósticas. Rev Fac Med. 2016;64(4):769-75.

3. Zárate A, Saucedo R, Basurto L, Hernández M. El nuevo enfoque hacia la diabetes gestacional. Rev Med Inst Mex Seguro Soc. 2011;49(1):1-3.

4. Bakris G, Blonde L, Boulton A. Standards of medical care in diabetes. 2018 J Clin Appl Res Educ. 2018;41(1):S1-159.

5. Arroyo L, Burbaro J. Diabetes y pie diabético: una problemática mundial abordada desde la fisioterapia. Rev Colomb Endocrinol Diabetes y Metab. 2019;6(3):199-208.

6. Márquez G. Consenso Latinoamericano de Diabetes y Embarazo. Asoc Latinoam Diabetes. 2007;1-14.

7. Instituto Nacional de Salud Pública. Encuesta nacional de salud y nutrición de medio camino 2016. Informe Final de Resultados. 2016.

8. Gastaldelli A, Gaggini M, De Defronzo R. Role of adipose tissue insulin resistance in the natural history of type 2 diabetes: results from the San Antonio metabolism study. Diabetes. 2017;66:815-22.

9. Atégbo M, Grissa A, Hichami K, Dramane L, Mountairou K, Miled A, et al. Modulation of adipokines and cytokines in gestational diabetes and macrosomia. J Clin Endocrinol Metab. 2006;91(10):4137-43.

10. Roll J, González N. Diabetes y obesidad: estudio en un área de salud. Rev Cubana Med Gen Integr. 2005;21,5-6.

11. de Gennaro G, Palla G, Battini L, Simoncini T, Prato S, Bertolotto A, et al. The role of adipokines in the pathogenesis of gestational diabetes mellitus. Gynecol Endocrinol. 2019;35(9):737-51.

12. Jayabalan N, Nair S, Nuzhat Z, Rice G, Zuñiga F, Lappas M. Cross talk between adipose tissue and placenta in obese and gestational diabetes mellitus pregnancies via exosomes. Front Endocrinol (Lausanne). 2017;8:239.

13. Benton M, Danielson K, Jones A, Macartney D, Das S, Ziegler O, et al. miRNA signatures of insulin resistance in obesity. Obesity. 2017;25(10):1734-44.

14. Samad F, Yamamoto K, Pandey M, Loskutoff D. Elevated expression of transforming growth factor- 3 in adipose tissue from obese mice. Mol Med. 1997;3(1):37-48.

15. Saleh J, Wahab R, Farhan H, Al-amri I, Cianflone K. Plasma levels of acylation-stimulating protein are strongly predicted by waist/hip ratio and correlate with decreased LDL size in men. ISRN Obes. 2013;(2013):1-6.

16. Goralski K, McCarthy T, Hanniman E, Zabel B, Parlee S, Muruganandan S, et al. Chemerin, a novel adipokine that regulates adipogenesis and adipocyte metabolism. J Biol Chem. 2007;282(38):28175-88.

17. Arana J, Carbó R, Hernández A, Sánchez F, Espinosa R. Omentina: papel en la resistencia a la insulina, inflamación y protección cardiovascular. Arch Cardiol Méx. 2015;86(3):233-43.

18. González F, Chávez A, Ramírez M, Pérez E, Moreno J, Saucedo M, et al. Quemerina en síndrome metabólico. El Residente. 2015;10(3):125-31.

19. van Poppel M, Ulrich D, Schest E, Hirschmugl B, Lang U, Wadsack C, et al Cord blood chemerin: differential effects of gestational diabetes mellitus and maternal obesity. Endocrinol Clin. 2013;80(1)65-72.

20. Zarini GG, Exebio JC, Gundupalli D, Nath S, Huffman FG. Hypertension, poor glycemic control, and microalbuminuria in Cuban Americans with type 2 diabetes. Int J Nephrol Renovasc Dis. 2011;(4):35-40.

21. Sanchez J, López D, Pinzón A, Sepúlveda A. Adipocinas y síndrome metabólico: múltiples facetas de un proceso fisiopatológico complejo. Rev Colomb Cardiol. 2010;17(4):167-76

22. De Souza C, Yang R, Lee M, Glynn N, Yu D, Pray J, et al. Omentin plasma levels and gene expression are decreased in obesity. Diabetes. 2007;56(6):1655-61.

23. Bork-Jensen B, Baun A, Bang-Bertelsen C, Grunnet L, Pociot F, Beck-Nielsen $H$, et al. Genetic versus non-genetic regulation of miR-103, miR-143 and miR-483-3p expression in adipose tissue and their metabolic implications - A twin study. Genes (Basel). 2014;5(3):508-17.

24. Pfau D, Fasshauer M, Stepan H, Drynda K, Lössner U, Verlohren M, et al Circulating levels of the adipokine chemerin in gestational diabetes mellitus. Horm Res Paediatr. 2010;74(1):56-61.

25. Yang X, Quan X, Lan Y, Ye J, Wei Q, Yin X, et al. Serum chemerin level during the first trimester of pregnancy and the risk of gestational diabetes mellitus. Gynecol Endocrinol. 2017;33(10):770-3.

26. Cheon D, Kang J, Lee S, Ihm S, Lee E, Choi M, et al. Serum chemerin levels are associated with visceral adiposity, independent of waist circumference, in newly diagnosed type 2 diabetic subjects. Yonsei Med J. 2017;58(2):319-25.

27. Panayoula C, Panagiotis H, Konstantinos P, Eirini M, George S, Sotirios A, et al. Circulating adipokines and mRNA expression in adipose tissue and the placenta in women with gestational diabetes mellitus. Peptides. 2018;101:157-66.

28. Barker G, Lim R, Georgiou HM, Lappas M. Omentin-1 is decreased in maternal plasma, placenta and adipose tissue of women with pre-existing obesity. PLoS One. 2012;7(8):1-8.

29. Abell SK, Shorakae S, Harrison C, Hiam D, Moreno A, Stepto N, et al. The association between dysregulated adipocytokines in early pregnancy and development of gestational diabetes. Diabetes Metab Res Rev. 2017;33(8):1-20.

30. Abell SK, Teede H, Boyle J, Stepto N, De Courten B, Harrison CL, et al. Role of serum biomarkers to optimise a validated clinical risk prediction tool for gestational diabetes. Aust New Zeal J Obstet Gynaecol. 2019:59(2):251-7.

31. Carreras-Badosa G, Bonmatí A, Ortega F, Mercader J, Guindo M, Torrents D, et al. Altered circulating miRNA expression profile in pregestational and gestational obesity. J Clin Endocrinol Metab. 2015;100(11):E1446-56.

32. Moher, D. Preferred Reporting Items for Systematic Reviews and MetaAnalyses: The PRISMA. Annals of Internal Medicine. 2009;151(4), 264. doi: 10.7326 / 0003-4819-151-4-200908180-00135.

33. El-Mesallamy HO, El-Derany MO, Hamdy NM. Serum omentin-1 and chemerin levels are interrelated in patients with type 2 diabetes mellitus with or without ischaemic heart disease. Diabet Med. 2011;28:1194-200.

34. American Diabetes Association. Classification and diagnosis of diabetes: standards of medical care in diabetes -2019. Diabetes Care. 2019;42(1):13-28

35. Almeida E, Sabino C, Galváo I, Silva A, Grande I. Razón entre grasa visceral y subcutánea como predictor de alteraciones cardiometabólicas. Rev Chil Nut. 2018;45(1):28-36

36. Tan B, Adya R, Farhatullah S, Lewandowski K, Hare P, Lehnert H, et al. Omentin-1, a novel adipokine, is decreased in overweight insulin-resistant women with polycystic ovary syndrome. Diabetes. 2008;57:801-8.

37. Coelho M, Oliveira T, Fernandes R. State of the art paper biochemistry of adipose tissue: an endocrine organ. Arch Med Sci. 2013.

38. Bozaoglu K, Curran J, Stocker C, Zaibi M, Segal D, Konstantopoulos N, et al. Chemerin, a novel adipokine in the regulation of angiogenesis. J Clin Endocrinol Metab. 2010;95(5):2476-85.

39. Álvarez G, Delgado J. Diseño de estudios epidemiológicos. I. El estudio transversal: tomando una fotografía de la salud y la enfermedad. Bol Clin Hosp Infant Edo Son. 2015;32(1):26-34

40. International Association of Diabetes and Pregnancy Study Groups Consensus Panel. International Association of Diabetes Pregnancy study Groups recommendations on the diagnosis and classification of hyperglycemia in pregnancy. Diabetes Care. 2010;33(3):662-82.

41. Hulley S, Cummings S, Browner W, Grady D, Newman T. Delineando a pesquisa clínica. 4a edición. Porto Alegre: Artmed. 2015.

42. Martínez D. Qualis periódicos: el sistema brasileño de evaluación de revistas. Anuario ThinkEPI. 2019,13:1-12. 